\title{
Lessons from Vibrio Pathogen and the Comparative Study of Vaccines Developed
}

\author{
Tania Rahman ${ }^{1,2^{*}}$, Md Ferdous Seraj ${ }^{3}$, Silvia Buroni ${ }^{4}$ \\ ${ }^{1}$ Department of Biochemistry and Molecular Biology, Bio21 Molecular Science and Biotechnology Institute, University of \\ Melbourne, Melbourne, Australia \\ ${ }^{2}$ Department of Microbiology and Immunology, University of Melbourne at the Peter Doherty Institute for Infection and \\ Immunity, Melbourne, Australia \\ ${ }^{3}$ School of Civil, Environmental and Chemical Engineering, RMIT University, Melbourne, Australia \\ ${ }^{4}$ Department of Biology and Biotechnology, University of Pavia, Pavia, Italy \\ Email: ^tania.rahman@du.ac.bd
}

How to cite this paper: Rahman, T., Seraj, M.F. and Buroni, S. (2018) Lessons from Vibrio Pathogen and the Comparative Study of Vaccines Developed. Advances in Microbiology, 8, 950-964.

https://doi.org/10.4236/aim.2018.812064

Received: September 25, 2018

Accepted: November 30, 2018

Published: December 17, 2018

Copyright (c) 2018 by authors and Scientific Research Publishing Inc. This work is licensed under the Creative Commons Attribution International License (CC BY 4.0).

http://creativecommons.org/licenses/by/4.0/

\begin{abstract}
Cholera continues to be one of the most common causes of morbidity and mortality among children and adults in developing countries. Vaccine against cholera is an approach in the control of this epidemic and pandemic disease. From the development of very early oral cholera vaccine, advances in vaccine development documented due to a good illustration of the epidemiology, outbreak strategy, and pathophysiology of the disease causing pathogen. The newer-generation oral cholera vaccines are safe and guarantee a high level of protection during outbreak settings for several years. Yet infants and young children in developing countries are hyporesponsive to vaccines and show poor protection against cholera. In this review, we survey and analyse our current knowledge on the etiology of cholera, its clinical manifestation, global epidemiology and elaborate the vaccine candidates, which are effective against the pathogen and the corresponding immune responses to the available vaccines. These reviews comprehensively cover the salient features of recent discoveries related to Vibrio cholerae virulence, past and present vaccine candidates and their advantages and disadvantages with their development strategies. We believe that the advances that have been included in this review will give a comprehensive insight to the prevention and control of cholera outbreaks and development of effective cholera vaccines.
\end{abstract}

\section{Keywords}

Vibrio cholerae, Serogroup O1, O139, Cholera Toxin, LPS, Vibriocidal Antibodies, IgA Antibodies, Heat-Killed Whole Cell Vaccine, Live Attenuated Oral Vaccine 


\section{Introduction}

Cholera is a waterborne and highly infectious disease that has caused devastating outbreaks in most parts of the world [1]. It is an acute watery diarrhoea caused by the Gram-negative bacillus Vibrio cholera, especially of the O1 serogroup [2]. If untreated, the drastic intestinal fluid loss caused by the pathogen can often lead to death [3]. Globally, 2.8 million cases of cholera occur each year, resulting in an estimated 91,000 deaths [4]. In spite of simple and widely accessible oral rehydration treatment, small children and adults are particularly vulnerable to the extreme dehydration of severe cholera [5]. Case-fatality rates may exceed $50 \%$ for those without treatment and be less than $1 \%$ among adequately treated individuals. The establishment of adequate personal hygiene, food safety and sanitation is important for control of cholera. However, in the short term, drastic improvements in these fields are difficult to achieve in areas where cholera is endemic. In the meantime, there is an urgent need for effective vaccines as an additional public health tool for cholera prevention. Effective vaccines are critical requirements for countries of Asia and Africa where the disease is endemic and is also seen in epidemics and during natural calamities [6]. It causes substantial morbidity and mortality in children between 2 to 5 years of age [7]. In fact, the disease has spread across the boundaries of Asia and Africa and epidemics of cholera have occurred in Central and South America with imported cases in other developed countries [8]. Here, we have done a comprehensive study of the disease pathogen, body's immune responses towards it and vaccine effectiveness in conjunction with combining information on the vaccination status and disease outcomes from cholera endemic areas.

\section{Vibrio cholerae, the Causative Bacteria}

The genus Vibrio comprises Gram-negative straight or curved rods belonging to the family Vibrionaceae [9]. In many aspects, vibrios are related to enteric bacteria. Vibrios are different from other enteric bacteria due to their oxidase-positive attributes and their motility using polar flagella [2]. Of all the vibrios which are clinically noteworthy to humans, Vibrio cholerae, the causative pathogen of cholera, is the most important.

The name, $V$. cholerae originates from the Greek words meaning, "flow of bile" [10]. V. cholerae is a Gram-negative, rod-shaped, mainly water-borne bacterium carrying a single polar flagellum [11]. V. cholerae can be classified into serogroups based upon polysaccharides of the somatic $(\mathrm{O})$ antigen [12]. It is not until 1992 while most of the epidemics of cholera were transmitted by Vibrio cholerae of the O1 serogroup [13]. Three serotypes and two biotypes of $V$. cholerae $\mathrm{O} 1$ have been described [14] [15]. Serotyping is based on somatic antigens and biotyping is according to specific phenotypic properties [16]. Ogawa (somatic antigens $A$ and $C$ ), Inaba (A and B), and Hikojima (A, B and C) designate the serotypes [17]. E1 Tor and classic designate the biotypes. The E1 Tor biotype, originally isolated as an avirulent strain in 1905, has evolved to greater vi- 
rulence and is responsible for the current pandemic [18]. In 1992, a new serogroup - a genetic derivative of the E1 Tor biotype-emerged in Bangladesh and caused an extensive epidemic [19]. It was later isolated from other parts of Asia and has been termed as $V$. cholerae O139 [20].

\subsection{Global Epidemiology of Cholera}

Cholera usually occurs in large epidemics or pandemics and in the $19^{\text {th }}$ century pandemics frequently originated from the Ganges delta in India [21]. There have been seven pandemics of cholera in recorded history [18]. Despite the etiological agents of the first four pandemics are not identified since they occurred during the time before these agents could be known, the last three pandemics are recognized to be caused by $V$. cholerae serogroup $\mathrm{O} 1$ [22]. The current, seventh pandemic caused by $V$. cholerae O1 E1 Tor originated in Indonesia in 1961 and spread rapidly through most of Asia into eastern Europe [23]. In 1970, this biotype was hosted into West Africa, where it spread briskly and is now endemic in many African countries. In 1991, it was reintroduced in to Peru (South American continent), where it had been absent for over 100 years [24]. Another serogroup, $V$. cholerae O139, was discovered as being the cause of cholera epidemics in India and Bangladesh in 1992 and since then it has spread to eleven other countries in South East Asia [21]. In 1992, in Bangladesh during a 12-week period, there were approximately 220,000 cases of cholera caused by serotype O139, with over 8000 deaths, more deaths than in all of Latin America the same year [25].

\subsection{Clinical Manifestation of Cholera}

Cholera is one of the most rapidly fatal illnesses known till date, in its extreme manifestation [26]. A healthy person may become hypotensive within an hour of the onset of symptoms and may die within 2 - 3 hours if no treatment is provided. Universally, the disease progresses from the first liquid stool to shock in 4 to 12 hours, with death following in 18 hours to several days [3]. The clinical description of cholera begins with sudden onset of massive diarrhea [27]. The patient could lose gallons of protein-free fluids and associated electrolytes, bicarbonates and ions within a couple of hours [28]. This fluid loss ultimately leads to dehydration, acidosis and shock. The watery diarrhea is dappled with bits of mucus and epithelial cells ("rice-water stool") and comprises huge numbers of vibrios [29]. The loss of ions particularly potassium could sometimes result in cardiac complications and circulatory failure. If untreated, cholera frequently results in mortality rates around 50\% - 60\% [30].

\subsection{Cholera Toxin, the Main Culprit}

Koch, who identified $V$. cholerae as the causative agent of cholera, had in 1887 already proposed that the disease was toxin-mediated but it was not until 1959 that the Indian scientists De and Dutta [31] convincingly demonstrated the exis- 
tence of cholera toxin [32]. It is now established that $V$. cholerae adheres to and colonizes the small intestine and secrete cholera toxin-that binds to receptors on the mucosal cells [9]. Cholera toxin (CT) is a protein that is composed of five receptor binding B subunits surrounding one catalytic A subunit [33]. While the $B$ subunits are aggregated in a ring by tight, non-covalent interactions [34], the A subunit is linked to and partially inserted in the B ring through weaker non-covalent bonds [35].

\subsection{Mode of Action of Cholera Toxin}

$V$. cholerae affects the small intestine through its secreted cholera toxin (CT) [36]. It is now known that the membrane receptor for cholera toxin is a specific ganglioside (monosialosyl ganglioside, GM1), which is ubiquitously distributed in the cell membrane of normal mammalian cells [37]. The mode of action of cholera toxin is summarized in Figure 1.

(1) When cholera toxin is secreted from the bacteria, it binds to the epithelial cell known as "enterocyte" in the lumen of infected intestine through the interaction of the pentameric B subunit of the toxin with the GM1 ganglioside receptor on the intestinal cell, triggering endocytosis of the toxin. (2) Next, the A subunit proteolytically cleaves into A1 and A2 peptides in order for A1 to become an active enzyme. Once inside the enterocyte, the enzymatic A1 fragment

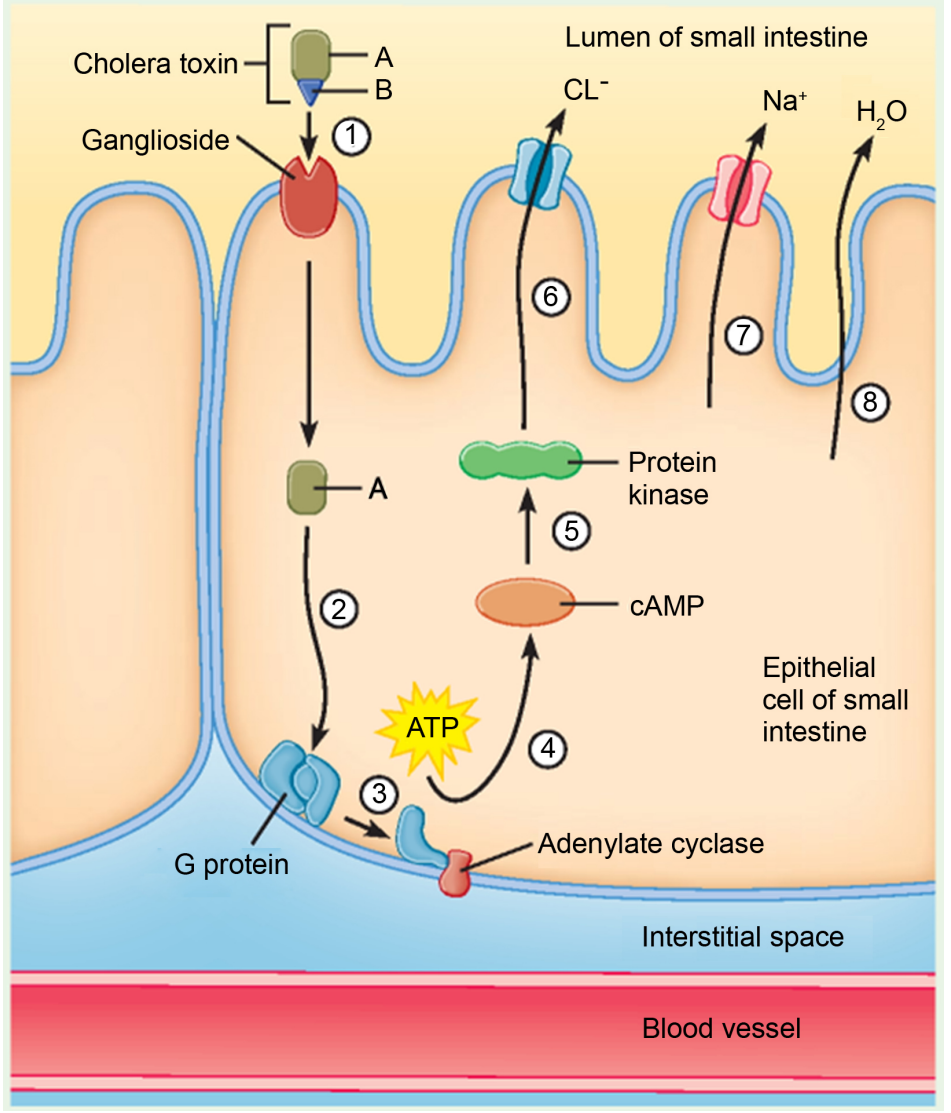

Figure 1. Mode of action of Cholera toxin (Adapted from [38]). 
of the toxin A subunit enters the cytosol, where it activates the $G$ protein $G_{s} \alpha$ through an ADP-ribosylation reaction. (3) This ADP-ribosylation acts to lock the G protein in its GTP-bound form, thereby blocking their inherent GTPase activity. (4) This leads to constitutive activation of adenylate cyclase and the rapid elevation of cAMP levels from ATP inside the cells. (5) The high cAMP levels, in turn, phosphorylates and then activates cAMP-dependent "protein kinase A". (6) (7) (8) Phosphorylated Protein kinase A then phosphorylates and hence activates proteins involved in the secretion of chloride ions, sodium ions and water. This dramatic efflux of ions and water from infected enterocytes leads to watery diarrhoea.

\subsection{Immune Response to Vibrio cholerae}

Epidemiological studies of cholera in endemic areas [39] and in human volunteers [40] have demonstrated that disease caused by $V$. cholerae gives rise to long-lasting protective immunity. The incidence of cholera disease is decreased with increasing age and recurrences are extremely rare in endemic areas [41] [42]. Cholera infection is associated with a rise in titer of a variety of circulating antibodies including vibriocidal antibodies [43] [44] and antibodies directed against cholera toxin (CT) [45] and cell wall lipopolysaccharide (LPS) [46].

Antibacterial antibodies, which develop in response to LPS, may protect against colonization with $V$. cholerae while antibody to CT may protect against disease in persons who are already colonized with $V$. cholerae [47] [48].

After natural infection by $V$. cholerae, circulating antibodies can be detected against several cholera antigens including $\mathrm{CT}$, somatic $(\mathrm{O})$ antigens, toxin co-regulated pilus (TCP) and mannose-sensitive hemagglutinin (MSHA) [49] [50]. These antibodies are also raised by parenteral injection of antigens as vaccine components. The early systemic response to somatic antigens is of the IgM class [51]. Subsequent challenges by either natural or vaccine antigens tend to induce a switch to IgG class antibodies [52]. However, in terms of protective immunity, the mucosal immune response is the most important. The intestinal IgA antibodies are the major immunoglobulin in mucosal immune response [53] [54]. These antibodies are produced locally in the intestinal mucosa and secreted onto the gut mucosal surface. The antibodies are mainly directed against bacterial components including $\mathrm{CT}$, and protection is by inhibiting bacterial colonization [54] and multiplication and by blocking toxin action.

The important action of antibodies is the one directed against Vibrio $\mathrm{O}$ antigens and these are considered "vibriocidal" antibodies because they lyse $V$. cholerae cells in the presence of complement and serum components [55]. Vibriocidal antibodies which return to the baseline 2 to 7 months after the onset of clinical illness reach a peak 8 to 10 days after the infection [56]. Their presence correlates with resistance to infection; however, they may not be the mediators of this protection and their role in natural infection is unclear.

Circulating anti-CT antibodies may also confer short protection, albeit not at 
the relatively low level induced by natural infection [57]. Adding the B subunit of CT to an oral vaccine stimulates mucosal formation of intestinal IgA antitoxin and contributes to protection for up to 9 months after vaccination [58].

\section{Cholera Vaccine Candidates-Past and Present}

\subsection{Cholera Vaccines to Prevent against Vibrio cholerae 01 Infection}

Enteric infections resulting in diarrhoeal disease from Vibrio cholerae remain a leading global health problem [59]. Although much sought for about a hundred years, since the identification of $V$. cholerae $\mathrm{O} 1$ as a causative agent of cholera, an effective and protective cholera vaccine still evades mankind [32]. The vaccine used until 20 to 30 years ago and licensed in the USA was a heat-killed whole cell parenteral vaccine, which gave short-lived protection only in adults [60]. The vaccine is no longer used because it has only limited efficacy $(\sim 50 \%)$ and duration of protection hardly exceeds 6 months [61]. Moreover, this licensed vaccine often causes pain and adverse effects at the injection site.

It has become increasingly recognized that for enteric diseases, especially those caused by bacteria, which are noninvasive, systemic immunization is not sufficient for inducing protective immunity [62]. As more knowledge of the mucosa-associated lymphoid tissue (MALT) is emerging, it has become obvious that an effective vaccine must stimulate the gut-associated lymphocytes to produce specific antibodies in the gut to inhibit colonization of the pathogen [63]. The vaccine should be capable of inducing both antibacterial and antitoxic immunity [64]. The vaccine should contain components which can stimulate memory cells comparable to natural infections. Their inadequacy to induce an appropriate memory response involves both B and T lymphocytes [65] [66].

From the beginning of the 1980s till today, much emphasis was placed by various groups of scientists in the design of an effective oral cholera vaccine that will be able to prevent disease caused by $V$. cholerae O1 [67]. Much success has been achieved in this area as a result of work carried out by two different groups of researchers [68]. Two licensed vaccines have emerged recently, which have been field-tested. Both are oral vaccines, but based on two opposite concepts: 1) One is killed, whole cell vaccine, containing a mixture of $\mathrm{O} 1$ bacteria of both Classical and E1 Tor biotypes and Ogawa and Inaba serotypes. $1 \mathrm{mg}$ of recombinant B subunit of cholera toxin (rBS) is added [61] and is given in two oral doses. An extensive field-trial has been carried out in Bangladesh (Clemens et al., 1986) and this has shown that it gives $85 \%$ protection in the first 6 months and about $60 \%$ protection over a period of 2 - 3 years. Field trials have also been carried out in Peru [69] (Concha et al., 1995). Recently, the most widely used oral killed cholera vaccine is Dukoral vaccine consisting of recombinantly produced cholera toxin B subunit (CTB) and inactivated $V$. cholerae $\mathrm{O} 1$ whole cells [70].

2) The second vaccine which has been licensed, is new generation live vaccine 
based on genetic engineering [71]. A strain of $V$. cholerae (Vibrio cholerae O1, classical, Inaba, strain 5698) has been attenuated so that the cholera toxin A subunit gene has been deleted [72]. As a result, the mutant strain does not produce cholera toxin. After extensive molecular biological studies, the strain has been sufficiently attenuated not to cause reactogenicity in vaccines. This vaccine candidate strain, CVD103HgR, is in addition resistant to mercury and therefore can be differentiated from naturally occurring O1 cholera strains [57] [71]. It has been tested in volunteers in the USA, Peru [63], and a large field-test has been carried out in Indonesia. The vaccine is given in a single dose. It has been developed in the USA and manufactured in Switzerland. Another vaccine named Shanchol has been prequalified by the WHO and is a formulation of killed $V$. cholerae cells (both V. cholerae O1 and O139). It is manufactured by Shantha Biotechnics of India, a subsidiary of the French pharmaceutical company Sanofi-Aventis [60]. It is a two-dose oral vaccine and has been established by a group of researchers from Sweden and South Korea, and its preliminary studies were carried out in Vietnam [73].

Killed cholera vaccines are safe since the fear of reacquiring genetic elements from virulent strains in the environment and in the host gut does not arise [55] [57]. On the other hand, the live cholera vaccine may be more immunogenic because it is able to colonize the gut, penetrate the M cells of the Peyer's patches and possibly stimulate better the natural course of events of the virulent $V$. cholerae organisms [74].

A second live vaccine candidate, Peru 15 is a Vibrio cholerae O1 E1 Tor, Inaba strain that has been engineered to be nontoxinogenic [75] (it lacks the $c t x A$ and rtx $A$ genes, which encode cholera toxin A subunit and the RTX toxin, respectively), nonrecombanitorial (it lacks the $\operatorname{rec} A$ gene and the attachment site for the CTX phage), nonmotile, and $c t x B$ positive (it makes the immunogenic, nontoxic CTB subunit) [76] [77]. It has been found to be safe and immunogenic against Vibrio cholerae O1 E1 Tor cholera in North American volunteers in experimental challenge studies [78]. This live attenuated oral vaccine was studied for safety and immunogenicity in Bangladeshi adults and infants [68].

\subsection{Bivalent Cholera Vaccine to Protect against Vibrio cholerae 01 and 0139}

The progress made in the late 1980s in the development of an effective cholera vaccine has been jeopardized when in October 1992 a new strain of Vibrio cholerae serogroup O139 emerged in India and Bangladesh as an epidemic strain [21] [79]. Efforts to make a cholera vaccine that can protect against both $\mathrm{O} 1$ and O139 cholera has led to the development of the bivalent whole-cell O1/O139 cholera vaccine by Swedish scientists who had developed the field-tested killed O1 cholera vaccine [57] [61]. This vaccine is basically composed of the field-tested and licensed $\mathrm{O} 1$ vaccine plus $\mathrm{rBS}$ which, in addition, contains $5 \times 10^{10}$ organisms of $V$. cholerae $\mathrm{O} 139$ [2]. Safety and immunogenicity studies on bivalent vaccine have been carried out simultaneously in Sweden, USA, Finland, and Bangladesh. 
In addition, live vaccine candidates, such as Bengal 15, has been developed and is being evaluated in volunteers [80]. A live oral carrier-based O139 vaccine has been genetically engineered in the CVD103HgR strain [75]. The vaccine candidates, $\mathrm{CH} 25$ or $\mathrm{CH} 26$, express short oligopolysaccharides as well as lipopolysaccharide of $V$. cholerae $\mathrm{O} 139$, presumably the key protective antigens for prevention of O139 cholera [81] [82].

\subsection{Immune Response to Killed Oral Cholera Vaccines}

Killed oral cholera vaccines have been designed to stimulate mucosal immune responses in the intestine similar to that induced by natural exposure [57] [83]. Animal data showed that oral whole inactivated bacteria induce anti-bacterial antibodies and that of the cholera toxin B subunit induced antitoxic antibodies [84]. These antibodies gave synergistic protection against subsequent infection with cholera (Svennerholm, 1976). Effective oral cholera vaccine contains both the inactivated whole bacteria and B subunit of the toxin.

With the whole-cell/B subunit vaccine, intestinal IgA responses are seen in most vaccines [85]. However, a rise in antitoxin is generally seen after the first dose, whereas an antibacterial response frequently requires two doses to produce [45]. As the vaccine stimulates local IgA antibodies and because there is evidence of a common mucosal immune system, titers of antibody in intestinal secretions have been examined after immunization [32]. Titer rises of IgA antitoxin and anti-LPS are frequently seen in intestinal secretions [46].

\subsection{Problems with Cholera Vaccines: Development Strategies}

An ideal vaccine is reasonably easy to outline, however, only limited real vaccines approach the ideal. On top of that, no vaccines exist for many organisms, for which a vaccine is the only faithful protective strategy in the foreseeable future [86]. An ideal vaccine: [64] 1) should prevent disease transmission, 2) should provide life-long immunity, 3) should be broadly protective against all variants of an organism, 4) should induce effective immunity rapidly, 5) should be effective in all vaccinated subjects, including infants and the elderly, 6) would not need to be administered by injection and 7) should be cheap, stable (no requirement for cold chain) and safe [87].

The outbreaks of cholera occur in a regular seasonal pattern in developing countries. In Bangladesh, an epidemic outbreak of cholera usually occurs twice in a year and the high-risk group is children [88]. Consequently, vaccines against cholera have to be designed and formulated that the immunization scheme, route of administration and dosage should be such that the vaccine does not interfere with the response in the host to the other bacteria [87].

\section{Concluding Remarks}

The described vaccines are dependent on the induction of a mucosal immune response for protection. Nevertheless, for all vaccines, practically long-lasting 
protection (memory) is a desirable objective despite requiring different types of immune response for protection [89]. The problems encountered in designing long-term memory response can be resolved by introducing components in the vaccine, which in natural disease, induce memory responses. Since acute watery diarrhoea caused by cholera is noninvasive, the vaccine candidates should be able to simulate the immune response by being taken up by the $\mathrm{M}$ cells, which are major sites for antigen uptake in the gut [62]. The introduction of CTB, which has mucosal immunopotentiating activity, has increased components which have adjuvant activity of CT, but lack the toxic properties. Since the adjuvant activity of $\mathrm{CT}$ is closely linked to the ADP-ribosylating action of the A subunit of CT, efforts are being made to make derivatives of $V$. cholerae strains which make cholera toxin A subunits inactive [33]. These strains, if sufficiently immunogenic and non-reactogenic, can be used as future vaccine candidates since they will have adjuvant capability as well as properties of inducing antitoxic immunity.

\section{Future Work}

Establishment of an adequate sanitation and potable-water system is the most complete way to prevent and limit the spread of cholera. The promotion of WASH (water, sanitation and hygiene) practices, the creation of rehydration centres, the use of antibiotics, and the training of health personnel could drastically reduce cholera-associated mortality [90]. The dual action of oral cholera vaccine play and WASH practices could reduce the intensity of morbidities in endemic areas. Further follow-up in our study will be required to ascertain the duration of protection conferred by recently developed cholera vaccines in children and adults.

\section{Acknowledgements}

This research was supported by the Italian Ministry of Education, University and Research (MIUR): Dipartimenti di Eccellenza Program (2018-2022), Department of Biology and Biotechnology, "L. Spallanzani”, University of Pavia (to SB).

\section{Conflicts of Interest}

The authors declare no conflict of interest that could be perceived to bias the work.

\section{References}

[1] Lipp, E.K., Huq, A. and Colwell, R.R. (2002) Effects of Global Climate on Infectious Disease: The Cholera Model. Clinical Microbiology Reviews, 15, 757-770. https://doi.org/10.1128/CMR.15.4.757-770.2002

[2] Finkelstein, R.A. (1996) Vibrio cholerae O1 and O139, and Other Pathogenic Vibrios. In: Baron, S., Ed., Medical Microbiology, 4th Edition, Chapter 24 (Cholera), Galveston, TX.

[3] Nelson, E.J., et al. (2009) Cholera Transmission: The Host, Pathogen and Bacterio- 
phage Dynamic. Nature Reviews Microbiology, 7, 693-702. https://doi.org/10.1038/nrmicro2204

[4] Ali, M., et al. (2015) Updated Global Burden of Cholera in Endemic Countries. PLoS Neglected Tropical Diseases, 9, e0003832. https://doi.org/10.1371/journal.pntd.0003832

[5] Brandt, K.G., Castro Antunes, M.M. and Silva, G.A. (2015) Acute Diarrhea: Evidence-Based Management. Jornal de Pediatria, 91, S36-S43. https://doi.org/10.1016/j.jped.2015.06.002

[6] Jutla, A., et al. (2013) Environmental Factors Influencing Epidemic Cholera. The American Journal of Tropical Medicine and Hygiene, 89, 597-607. https://doi.org/10.4269/ajtmh.12-0721

[7] Lanata, C.F., et al. (2013) Global Causes of Diarrheal Disease Mortality in Children <5 Years of Age: A Systematic Review. PloS ONE, 8, e72788. https://doi.org/10.1371/journal.pone.0072788

[8] Guthmann, J.P. (1995) Epidemic Cholera in Latin America: Spread and Routes of Transmission. The Journal of Tropical Medicine and Hygiene, 98, 419-427.

[9] Almagro-Moreno, S., Pruss, K. and Taylor, R.K. (2015) Intestinal Colonization Dynamics of Vibrio cholerae. PLoS Pathogens, 11, e1004787. https://doi.org/10.1371/journal.ppat.1004787

[10] Colwell, R.R. (1996) Global Climate and Infectious Disease: The Cholera Paradigm. Science, 274, 2025-2031. https://doi.org/10.1126/science.274.5295.2025

[11] Millet, Y.A., et al. (2014) Insights into Vibrio cholerae Intestinal Colonization from Monitoring Fluorescently Labeled Bacteria. PLoS Pathogens, 10, e1004405. https://doi.org/10.1371/journal.ppat.1004405

[12] Chatterjee, S.N. and Chaudhuri, K. (2003) Lipopolysaccharides of Vibrio cholerae. I. Physical and Chemical Characterization. Biochimica et Biophysica Acta, 1639, 65-79. https://doi.org/10.1016/j.bbadis.2003.08.004

[13] Villeneuve, S., et al. (1999) Immunochemical Characterization of an Ogawa-Inaba Common Antigenic Determinant of Vibrio cholerae O1. Microbiology, 145, 2477-2484. https://doi.org/10.1099/00221287-145-9-2477

[14] Alam, M., et al. (2006) Seasonal Cholera caused by Vibrio cholerae Serogroups O1 and $\mathrm{O} 139$ in the Coastal Aquatic Environment of Bangladesh. Applied and Environmental Microbiology, 72, 4096-4104. https://doi.org/10.1128/AEM.00066-06

[15] Son, M.S., et al. (2011) Characterization of Vibrio cholerae O1 El Tor Biotype Variant Clinical Isolates from Bangladesh and Haiti, Including a Molecular Genetic Analysis of Virulence Genes. Journal of Clinical Microbiology, 49, 3739-3749. https://doi.org/10.1128/JCM.01286-11

[16] Chen, C.H., Shimada, T., Elhadi, N., Radu, S. and Nishibuchi, M. (2004) Phenotypic and Genotypic Characteristics and Epidemiological Significance of $c t x^{+}$Strains of Vibrio cholerae Isolated from Seafood in Malaysia. Applied and Environmental Microbiology, 70, 1964-1972. https://doi.org/10.1128/AEM.70.4.1964-1972.2004

[17] Meeks, M.D., et al. (2004) Synthetic Fragments of Vibrio cholerae O1 Inaba O-Specific Polysaccharide Bound to a Protein Carrier Are Immunogenic in Mice but Do Not Induce Protective Antibodies. Infection and Immunity, 72, 4090-4101. https://doi.org/10.1128/IAI.72.7.4090-4101.2004

[18] Hu, D., et al. (2016) Origins of the Current Seventh Cholera Pandemic. Proceedings of the National Academy of Sciences of the United States of America, 113, E7730-E7739. https://doi.org/10.1073/pnas.1608732113 
[19] Nair, G.B., Faruque, S.M., Bhuiyan, N.A., Kamruzzaman, M., Siddique, A.K. and Sack, D.A. (2002) New Variants of Vibrio cholerae O1 Biotype El Tor with Attributes of the Classical Biotype from Hospitalized Patients with Acute Diarrhea in Bangladesh. Journal of Clinical Microbiology, 40, 3296-3299. https://doi.org/10.1128/JCM.40.9.3296-3299.2002

[20] Sinha, S., et al. (2002) Escalating Association of Vibrio cholerae O139 with Cholera Outbreaks in India. Journal of Clinical Microbiology, 40, 2635-2637. https://doi.org/10.1128/JCM.40.7.2635-2637.2002

[21] Faruque, S.M., et al. (2003) Emergence and Evolution of Vibrio cholerae O139. Proceedings of the National Academy of Sciences of the United States of America, 100, 1304-1309. https://doi.org/10.1073/pnas.0337468100

[22] Almagro-Moreno, S. and Taylor, R.K. (2013) Cholera: Environmental Reservoirs and Impact on Disease Transmission. Microbiology Spectrum, 1.

[23] Weill, F.X., et al. (2017) Genomic History of the Seventh Pandemic of Cholera in Africa. Science, 358, 785-789. https://doi.org/10.1126/science.aad5901

[24] Poirier, M.J., Izurieta, R., Malavade, S.S. and McDonald, M.D. (2012) Re-Emergence of Cholera in the Americas: Risks, Susceptibility, and Ecology. Journal of Global Infectious Diseases, 4, 162-171. https://doi.org/10.4103/0974-777X.100576

[25] Siddique, A.K., Zaman, K., Akram, K., Mutsuddy, P., Eusof, A. and Sack, R.B. (1994) Emergence of a New Epidemic Strain of Vibrio cholerae in Bangladesh. An Epidemiological Study. Tropical and Geographical Medicine, 46, 147-150.

[26] Dick, M.H., Guillerm, M., Moussy, F. and Chaignat, C.-L. (2012) Review of Two Decades of Cholera Diagnostics-How Far Have We Really Come? PLoS Neglected Tropical Diseases, 6, e1845. https://doi.org/10.1371/journal.pntd.0001845

[27] Keen, M.F. and Bujalski, L. (1992) The Diagnosis and Treatment of Cholera. The Nurse Practitioner, 17, 53-56.

[28] Chowdhury, F., Khan, A.I., Faruque, A.S.G. and Ryan, E.T. (2010) Severe, Acute Watery Diarrhea in an Adult. PLoS Neglected Tropical Diseases, 4, e898. https://doi.org/10.1371/journal.pntd.0000898

[29] Alexakis, L.C. (2017) Cholera-Rice Water Stools. The Pan African Medical Journal, 26, 147. https://doi.org/10.11604/pamj.2017.26.147.11874

[30] Tobin-D’Angelo, M., et al. (2008) Severe Diarrhea Caused by Cholera Toxin-Producing Vibrio cholerae Serogroup O75 Infections Acquired in the Southeastern United States. Clinical Infectious Diseases, 47, 1035-1040. https://doi.org/10.1086/591973

[31] Holmgren, J. (1981) Actions of Cholera Toxin and the Prevention and Treatment of Cholera. Nature, 292, 413-417. https://doi.org/10.1038/292413a0

[32] Ghose, A.C. (2011) Lessons from Cholera \& Vibrio cholerae. The Indian Journal of Medical Research, 133, 164-170.

[33] Bharati, K. and Ganguly, N.K. (2011) Cholera Toxin: A Paradigm of a Multifunctional Protein. The Indian Journal of Medical Research, 133, 179-187.

[34] Lahiani, A., Yavin, E. and Lazarovici, P. (2017) The Molecular Basis of Toxins' Interactions with Intracellular Signaling via Discrete Portals. Toxins, 9.

[35] Taylor, M., Curtis, D. and Teter, K. (2015) A Conformational Shift in the Dissociated Cholera Toxin A1 Subunit Prevents Reassembly of the Cholera Holotoxin. Toxins, 7, 2674-2684. https://doi.org/10.3390/toxins7072674

[36] Guichard, A., et al. (2013) Cholera Toxin Disrupts Barrier Function by Inhibiting 
Exocyst-Mediated Trafficking of Host Proteins to Intestinal Cell Junctions. Cell Host \& Microbe, 14, 294-305. https://doi.org/10.1016/j.chom.2013.08.001

[37] Cuatrecasas, P. (1973) Gangliosides and Membrane Receptors for Cholera Toxin. Biochemistry, 12, 3558-3566. https://doi.org/10.1021/bi00742a032

[38] Stanfield, C.L. (2017) Principles of Human Physiology. Pearson Global Edition.

[39] Holmgren, J. and Svennerholm, A.M. (1977) Mechanisms of Disease and Immunity in Cholera: A Review. The Journal of Infectious Diseases, 136, S105-S112. https://doi.org/10.1093/infdis/136.Supplement.S105

[40] Levine, M.M., et al. (1979) Immunity of Cholera in Man: Relative Role of Antibacterial versus Antitoxic Immunity. Transactions of the Royal Society of Tropical Medicine and Hygiene, 73, 3-9. https://doi.org/10.1016/0035-9203(79)90119-6

[41] Stoll, B.J., Glass, R.I., Huq, M.I., Khan, M.U., Banu, H. and Holt, J. (1982) Epidemiologic and Clinical Features of Patients Infected with Shigella Who Attended a Diarrheal Disease Hospital in Bangladesh. The Journal of Infectious Diseases, 146, 177-183. https://doi.org/10.1093/infdis/146.2.177

[42] Glass, R.I., et al. (1982) Endemic Cholera in Rural Bangladesh, 1966-1980. American Journal of Epidemiology, 116, 959-970. https://doi.org/10.1093/oxfordjournals.aje.a113498

[43] Patel, S.M., et al. (2012) Memory B Cell Responses to Vibrio cholerae O1 Lipopolysaccharide Are Associated with Protection against Infection from Household Contacts of Patients with Cholera in Bangladesh. Clinical and Vaccine Immunology, 19, 842-848. https://doi.org/10.1128/CVI.00037-12

[44] Jayasekera, C.R., et al. (2008) Cholera Toxin-Specific Memory B Cell Responses Are Induced in Patients with Dehydrating Diarrhea Caused by Vibrio cholerae O1. The Journal of Infectious Diseases, 198, 1055-1061. https://doi.org/10.1086/591500

[45] Leung, D.T., Chowdhury, F., Calderwood, S.B., Qadri, F. and Ryan, E.T. (2012) Immune Responses to Cholera in Children. Expert Review of Anti-Infective Therapy, 10, 435-444. https://doi.org/10.1586/eri.12.23

[46] Bishop, A.L., Schild, S., Patimalla, B., Klein, B. and Camilli, A. (2010) Mucosal Immunization with Vibrio cholerae Outer Membrane Vesicles Provides Maternal Protection Mediated by Antilipopolysaccharide Antibodies That Inhibit Bacterial Motility. Infection and Immunity, 78, 4402-4420. https://doi.org/10.1128/IAI.00398-10

[47] Wu, J.Y., Taylor, R.K. and Wade, W.F. (2001) Anti-Class II Monoclonal Antibody-Targeted Vibrio cholerae TcpA Pilin: Modulation of Serologic Response, Epitope Specificity, and Isotype. Infection and Immunity, 69, 7679-7686. https://doi.org/10.1128/IAI.69.12.7679-7686.2001

[48] Baranova, D.E., Levinson, K.J. and Mantis, N.J. (2018) Vibrio cholerae O1 Secretes an Extracellular Matrix in Response to Antibody-Mediated Agglutination. PLoS ONE, 13, e0190026. https://doi.org/10.1371/journal.pone.0190026

[49] Falero, G., et al. (2003) Production and Characterization of Monoclonal Antibodies to E1 Tor Toxin Co-Regulated Pilus of Vibrio cholerae. Hybridoma and Hybridomics, 22, 315-320.

[50] Tacket, C.O., et al. (1998) Investigation of the Roles of Toxin-Coregulated Pili and Mannose-Sensitive Hemagglutinin Pili in the Pathogenesis of Vibrio cholerae O139 Infection. Infection and Immunity, 66, 692-695.

[51] Gupta, R.K., Taylor, D.N., Bryla, D.A., Robbins, J.B. and Szu, S.C. (1998) Phase 1 Evaluation of Vibrio cholerae O1, Serotype Inaba, Polysaccharide-Cholera Toxin Conjugates in Adult Volunteers. Infection and Immunity, 66, 3095-3099. 
[52] Haney, D.J., Lock, M.D., Simon, J.K., Harris, J. and Gurwith, M. (2017) Antibody-Based Correlates of Protection against Cholera Analysis of a Challenge Study in a Cholera-Naive Population. Clinical and Vaccine Immunology, 24, e00098-17. https://doi.org/10.1128/CVI.00098-17

[53] Mantis, N.J., Rol, N. and Corthesy, B. (2011) Secretory IgA's Complex Roles in Immunity and Mucosal Homeostasis in the Gut. Mucosal Immunology, 4, 603-611. https://doi.org/10.1038/mi.2011.41

[54] Blanco, L.P. and Dirita, V.J. (2006) Antibodies Enhance Interaction of Vibrio cholerae with Intestinal M-Like Cells. Infection and Immunity, 74, 6957-6964. https://doi.org/10.1128/IAI.00905-06

[55] Son, M.S. and Taylor, R.K. (2011) Vibriocidal Assays to Determine the Antibody Titer of Patient Sera Samples. Current Protocols in Microbiology, 6A.3.1-6A.3.9. https://doi.org/10.1002/9780471729259.mc06a03s23

[56] Harris, A.M., et al. (2009) Antigen-Specific Memory B-Cell Responses to Vibrio cholerae $\mathrm{O} 1$ Infection in Bangladesh. Infection and Immunity, 77, 3850-3856. https://doi.org/10.1128/IAI.00369-09

[57] Bishop, A.L. and Camilli, A. (2011) Vibrio cholerae: Lessons for Mucosal Vaccine Design. Expert Review of Vaccines, 10, 79-94. https://doi.org/10.1586/erv.10.150

[58] Viret, J.F., et al. (1999) Mucosal and Systemic Immune Responses in Humans after Primary and Booster Immunizations with Orally Administered Invasive and Noninvasive Live Attenuated Bacteria. Infection and Immunity, 67, 3680-3685.

[59] Bump, J.B., Reich, M.R. and Johnson, A.M. (2013) Diarrhoeal Diseases and the Global Health Agenda: Measuring and Changing Priority. Health Policy and Planning, 28, 799-808. https://doi.org/10.1093/heapol/czs119

[60] Kabir, S. (2014) Critical Analysis of Compositions and Protective Efficacies of Oral Killed Cholera Vaccines. Clinical and Vaccine Immunology, 21, 1195-1205. https://doi.org/10.1128/CVI.00378-14

[61] Lopez, A.L., Gonzales, M.L.A., Aldaba, J.G. and Nair, G.B. (2014) Killed Oral Cholera Vaccines: History, Development and Implementation Challenges. Therapeutic Advances in Vaccines, 2, 123-136. https://doi.org/10.1177/2051013614537819

[62] Czerkinsky, C. and Holmgren, J. (2015) Vaccines against Enteric Infections for the Developing World. Philosophical Transactions of the Royal Society of London. Series B, Biological Sciences, 370, Article ID: 20150142. https://doi.org/10.1098/rstb.2015.0142

[63] Levine, M.M. (2010) Immunogenicity and Efficacy of Oral Vaccines in Developing Countries: Lessons from a Live Cholera Vaccine. BMC Biology, 8, 129. https://doi.org/10.1186/1741-7007-8-129

[64] Nascimento, I.P. and Leite, L.C. (2012) Recombinant Vaccines and the Development of New Vaccine Strategies. Brazilian Journal of Medical and Biological Research, 45, 1102-1111. https://doi.org/10.1590/S0100-879X2012007500142

[65] Esser, M.T., et al. (2003) Memory T Cells and Vaccines. Vaccine, 21, 419-430. https://doi.org/10.1016/S0264-410X(02)00407-3

[66] Sarkander, J., Hojyo, S. and Tokoyoda, K. (2016) Vaccination to Gain Humoral Immune Memory. Clinical \& Translational Immunology, 5, e120. https://doi.org/10.1038/cti.2016.81

[67] Shin, S., Desai, S.N., Sah, B.K. and Clemens, J.D. (2011) Oral Vaccines against Cholera. Clinical Infectious Diseases, 52, 1343-1349.

https://doi.org/10.1093/cid/cir141 
[68] Qadri, F., et al. (2005) Randomized, Controlled Study of the Safety and Immunogenicity of Peru-15, a Live Attenuated Oral Vaccine Candidate for Cholera, in Adult Volunteers in Bangladesh. The Journal of Infectious Diseases, 192, 573-579. https://doi.org/10.1086/432074

[69] Taylor, C.E. and Greenough 3rd, W.B. (1989) Control of Diarrheal Diseases. Annual Review of Public Health, 10, 221-244.

[70] Svennerholm, A.M. (2011) From Cholera to Enterotoxigenic Escherichia coli (ETEC) Vaccine Development. The Indian Journal of Medical Research, 133, 188-196.

[71] Michalski, J., et al. (1993) CVD110, an Attenuated Vibrio cholerae O1 El Tor Live Oral Vaccine Strain. Infection and Immunity, 61, 4462-4468.

[72] Baldauf, K.J., Royal, J.M., Hamorsky, K.T. and Matoba, N. (2015) Cholera Toxin B: One Subunit with Many Pharmaceutical Applications. Toxins, 7, 974-996. https://doi.org/10.3390/toxins7030974

[73] Anh, D.D., et al. (2007) Safety and Immunogenicity of a Reformulated Vietnamese Bivalent Killed, Whole-Cell, Oral Cholera Vaccine in Adults. Vaccine, 25, 1149-1155. https://doi.org/10.1016/j.vaccine.2006.09.049

[74] Miller, H., Zhang, J., Kuolee, R., Patel, G.B. and Chen, W. (2007) Intestinal M Cells: The Fallible Sentinels? World Journal of Gastroenterology, 13, 1477-1486.

[75] Liang, W., et al. (2003) Construction and Evaluation of a Safe, Live, Oral Vibrio cholerae Vaccine Candidate, IEM108. Infection and Immunity, 71, 5498-5504. https://doi.org/10.1128/IAI.71.10.5498-5504.2003

[76] Boyd, E.F. and Waldor, M.K. (1999) Alternative Mechanism of Cholera Toxin Acquisition by Vibrio cholerae: Generalized Transduction of CTXPhi by Bacteriophage CP-T1. Infection and Immunity, 67, 5898-5905.

[77] Lin, W., et al. (1999) Identification of a Vibrio cholerae RTX Toxin Gene Cluster That Is Tightly Linked to the Cholera Toxin Prophage. Proceedings of the National Academy of Sciences of the United States of America, 96, 1071-1076. https://doi.org/10.1073/pnas.96.3.1071

[78] Garcia, L., et al. (2005) The Vaccine Candidate Vibrio cholerae 638 Is Protective against Cholera in Healthy volunteers. Infection and Immunity, 73, 3018-3024. https://doi.org/10.1128/IAI.73.5.3018-3024.2005

[79] Waldor, M.K. and Mekalanos, J.J. (1994) Emergence of a New Cholera Pandemic: Molecular Analysis of Virulence Determinants in Vibrio cholerae O139 and Development of a Live Vaccine Prototype. The Journal of Infectious Diseases, 170, 278-283. https://doi.org/10.1093/infdis/170.2.278

[80] Coster, T.S., et al. (1995) Safety, Immunogenicity, and Efficacy of Live Attenuated Vibrio cholerae O139 Vaccine Prototype. The Lancet, 345, 949-952. https://doi.org/10.1016/S0140-6736(95)90698-3

[81] Hisatsune, K., Kondo, S., Isshiki, Y., Iguchi, T., Kawamata, Y. and Shimada, T. (1993) O-Antigenic Lipopolysaccharide of Vibrio cholerae O139 Bengal, a New Epidemic Strain for Recent Cholera in the Indian Subcontinent. Biochemical and Biophysical Research Communications, 196, 1309-1315. https://doi.org/10.1006/bbrc.1993.2395

[82] Attridge, S.R., Qadri, F., Albert, M.J. and Manning, P.A. (2000) Susceptibility of Vibrio cholerae O139 to Antibody-Dependent, Complement-Mediated Bacteriolysis. Clinical and Diagnostic Laboratory Immunology, 7, 444-450.

[83] Ogra, P.L., Faden, H. and Welliver, R.C. (2001) Vaccination Strategies for Mucosal 
Immune Responses. Clinical Microbiology Reviews, 14, 430-445.

https://doi.org/10.1128/CMR.14.2.430-445.2001

[84] Price, G.A., McFann, K. and Holmes, R.K. (2013) Immunization with Cholera Toxin B Subunit Induces High-Level Protection in the Suckling Mouse Model of Cholera. PLoS ONE, 8, e57269. https://doi.org/10.1371/journal.pone.0057269

[85] Pasetti, M.F., Simon, J.K., Sztein, M.B. and Levine, M.M. (2011) Immunology of Gut Mucosal Vaccines. Immunological Reviews, 239, 125-148. https://doi.org/10.1111/j.1600-065X.2010.00970.x

[86] Beverley, P.C. (2002) Immunology of Vaccination. British Medical Bulletin, 62, 15-28. https://doi.org/10.1093/bmb/62.1.15

[87] Bohles, N., Busch, K. and Hensel, M. (2014) Vaccines against Human Diarrheal Pathogens: Current Status and Perspectives. Human Vaccines \& Immunotherapeutics, 10, 1522-1535. https://doi.org/10.4161/hv.29241

[88] Sack, R.B., et al. (2003) A 4-Year Study of the Epidemiology of Vibrio cholerae in Four Rural Areas of Bangladesh. The Journal of Infectious Diseases, 187, 96-101. https://doi.org/10.1086/345865

[89] Burgers, W.A., et al. (2012) Measurements of Immune Responses for Establishing Correlates of Vaccine Protection against HIV. AIDS Research and Human Retroviruses, 28, 641-648.

[90] Taylor, D.L., Kahawita, T.M., Cairncross, S. and Ensink, J.H.J. (2015) The Impact of Water, Sanitation and Hygiene Interventions to Control Cholera: A Systematic Review. PLoS ONE, 10, e0135676. https://doi.org/10.1371/journal.pone.0135676 